\title{
Three-Dimensional Printing as a Potentially Effective Strategy for Improving the Outcomes in Microtia Reconstruction
}

\author{
OMAR M.A. EL-BAYOUMI, M.Sc.*; AHMED M. BAHAA EL-DIN, M.D.*; \\ FAYEZ SHAHATTO MAHMOUD, M.D.**; AHMED M. ZEINA, M.D.* and \\ MOSTAFA M. ABDELHALIM, M.D.* \\ The Departments of Plastic Surgery* and General Surgery**, Faculty of Medicine, Mansoura University
}

\begin{abstract}
Aim: The reconstruction of microtia cases is a challenging topic and auricular reconstruction is a unique area of facial plastic surgery where a wide array of reconstructive options often must be considered. The goal is to assess the use of three-dimensional (3D) printing to create a 3D model for use during reconstruction to achieve better aesthetic results.
\end{abstract}

Patients and Methods: This study included ten patients with microtia. Utilizing two stages of ear reconstruction, the authors used a $3 \mathrm{D}$ printed auricular model of the contralateral ear as an intraoperative guide. The Mansoura ear reconstruction scale was conceptualized and used by the authors to assess the final aesthetic outcomes.

Results: In total there were 10 patients with 11 affected auricles. Results were 6 patients with an excellent result, 3 patients with a very good result and one patient with a fair result according to Mansoura ear reconstruction scale.

Conclusions: The use of 3D printed models for microtia reconstruction resulted in an overall increase in aesthetic outcomes and increased patient satisfaction.

Key Words: Auricular model - Costal cartilage - 3D printing - Ear reconstruction microtia - Tissue expander.

\section{INTRODUCTION}

Auricular reconstruction is a unique area of facial plastic surgery where a wide array of reconstructive options often must be considered. The external ear is unique in its aesthetic role where the normal auricle often goes unnoticed; yet even a small irregularity can stand out and become conspicuous [1]. Throughout history, surgeons developed many different techniques and methods for total ear reconstruction. A number of flaps and procedures exist for that purpose [2].

Microtia is defined as a congenitally small or malformed auricle, usually with absent parts. This occurs when maldevelopment of first and second branchial arches occurs [3]. The microtia incidence ranges from 1 in 500 to 1 in 3000 live birth. Males are more affected. $80 \%$ of cases are unilateral and the right side is affected more [4]. The incidence is higher in Hispanics and Asians than in Whites and Blacks [5,6].

Costal cartilage is the gold standard autologous graft chosen for microtia reconstruction. It is abundant, accessible and easily fabricated [7]. A number of surgical techniques have been used for total ear reconstruction using costal cartilage, with each containing a number of different stages including Tanzer, Brent and Nagata [8].

The principle of tissue expansion is widely used in auricular reconstruction. Many surgeons used tissue expansion either in single stage or two stages, and they reported superior skin color and texture match, also, intact sensation of expanded skin [9].

Advances in three-dimensional (3D) imaging and printing technology have made it possible to produce more accurate templates for reconstructive surgery. In particular, surgeons in the field of craniofacial surgery have used 3D imaging and printing technology for preoperative planning, outcome \& evaluation [10]. This technology successfully helps to create parts like prosthetics, dental appliances and custom implants [11].

The 3D auricular template contributes considerably to the engraving and localization of the cartilage framework and the increased details allows for a better aesthetic outcome [12].

\section{PATIENTS AND METHODS}

This prospective study was conducted on ten patients with microtia. All patients underwent treatment between March 2020 to March 2021 at 
the Department of Plastic \& Reconstructive Surgery, Mansoura University. The study was approved by the Ethical Committee of Faculty of Medicine (MS.20.03.1062). Informed consent was obtained from the patients or their legal guardians explaining the procedure, side effects and possible complications in details.

Patients with microtia of different ages were included. Patients who refused surgery, patients with systemic conditions compromising wound healing and patients with mental instability were excluded.

\section{Operative stages:} ${ }^{1 \text { st }}$ stage:

The first stage was for implantation of the tissue expander under general anesthesia to gain sufficient tissue prior to the second stage. A rectangular tissue expander $\left(\right.$ Polytech $\left.{ }^{\circledR}\right)$ of appropriate size $(100 \mathrm{cc}$ in adults and $50 \mathrm{cc}$ in children) was used.

Skin and subcutaneous tissue were infiltrated with adrenaline $(1: 200000)$ to help in hemostasis prior to the skin incision. Skin was incised in occipital hair line. Postauricular skin was dissected in the subcutaneous plan to create a pocket $1 \mathrm{~cm}$ wider than the base of the tissue expander to be used (Fig. 1). The tissue expander was then inserted into this pocket, and the tissue expander's remote valve was inserted into the occipital scalp area. Prior to skin closure, a suction drain was inserted. After wound healing, the tissue expander was inflated on a weekly basis with normal saline $0.9 \%$, with the expander overinflated by $10-25 \%$ to gain enough tissue for the second stage (Fig. 2).

The $2^{\text {nd }}$ stage of reconstruction with costal cartilage was carried out 2-4 weeks after completing inflation to allow cutaneous flap maturity. The alginate imprint was taken from the normal ear prior to $2^{\text {nd }}$ stage of procedure and the $3 \mathrm{D}$ imprint was prepared.

\section{Template creation:}

First, a negative imprint of the normal ear was taken. For protection, a plastic frame was placed over the patient's normal ear and a piece of cotton was inserted into the external auditory canal, Cavex ${ }^{\circledR}$ CA37 fast set alginate was used. Alginate is poured into the plastic frame and allowed to dry for 10 minutes. The alginate model now provides us with a negative imprint of the normal ear (Fig. 3).

Next, the gypsum was then poured into the negative alginate mould. It was allowed to dry before being removed, providing us with a $3 \mathrm{D}$ model of the previously imprinted ear (Fig. 4A).

This three-dimensional model was then scanned with a three-dimensional scanner and imported into medical computer-aided design (CAD) software (Ceramill ${ }^{\circledR}$ mind). The CAD software was used to remove any artifacts or unnecessary impressions, and the final design was flipped to give us the desired rendering for the auricular reconstruction (Fig. 4B), this was done by taking the 3D scanned .stl render of the normal auricle and using the software which did a mirror image of this render, which gave us a new render of the desired auricle to be 3D printed. The final step was to print this design using a 3D printer (Creality® Dental 3D Printer), Polymethyl methacrylate (PMMA) was used as a printing material. The result was the final auricular model (Fig. 4C), which was sterilized and used intraoperatively during the second stage. For the patient with bilateral microtia, the ear imprint was taken from the normal ear of his brother.

\section{$2^{\text {nd }}$ stage:}

The second stage, which was performed under general anaesthesia, involved the harvesting of costal cartilage as well as the creation and insertion of the auricular framework (Fig. 5).

Skin incision on the same side of the affected ear's chest wall, from the seventh to the ninth rib, passing obliquely $1 \mathrm{~cm}$ superior to the costal margin. Subcutaneous undermining was used to expose the costal cartilage. To retract the skin and subcutaneous tissue while maintaining good exposure, retaining sutures were used. Using monopolar diathermy, a muscle splitting incision was made between the serratus anterior and rectus abdominis muscles. The sixth to ninth ribs were exposed. These ribs' costal cartilages were harvested subperichondrally, leaving the perichondrium intact. The junction of the $7^{\text {th }}$ and $8^{\text {th }}$ costal cartilages was preserved. Absorbable polyfilament sutures were used to close the perichondrium to preserve chest wall integrity and prevent deformity. The muscles were approximated with absorbable sutures, and the skin was closed over the suction drain with non-absorbable monofilament sutures.

\section{Creation of framework:}

The framework was fashioned guided by the 3D template (Fig. 5B,C). The framework was formed of three parts: the helix, the base, and the pad. The ninth cartilage formed the helix. The junction between $7^{\text {th }}$ and $8^{\text {th }}$ costal cartilages formed the base and carving of helix and antihelix 
over the base to increase the details. The sixth costal cartilage formed the base to achieve projection. Skin of the ear was incised as M-W flap and the lobule rotated to its new position. The vestigial cartilage was excised, and the tissue expander removed from the same incision after being deflated. The valve was removed using a small incision in occipital hair.

The cartilaginous framework was introduced in the skin pocket of expanded postauricular skin and was placed in its optimal position. The expanded skin flap was used to cover both the anterior and posterior aspects of the framework, two small suction catheters were inserted between skin flap and framework with one of them inserted between the helix and the antihelix and the other one inserted posteriorly to achieve skin coaptation and prevent post-operative hematoma formation (Fig. 6). Closure of the skin using non-absorbable monofilament sutures was done followed by light dressing. Removal of chest drain and one ear suction catheter on $4^{\text {th }}$ post-operative day and the other catheter on $7^{\text {th }}$ day.

\section{Follow-up:}

Patients were assessed using the Mansoura ear reconstruction scale, which is based on three scales: the photograph scale, the patient satisfaction scale, and the complication scale. A photograph scale (Table 1), in which patient photographs were evaluated by two independent surgeons to give a score from 0 to 3 , where 0 was a poor result and 3 was an excellent result, with an average score obtained. Patient satisfaction scale (Table 2), with each patient giving a score ranging from 0 to 3 , with 0 being unsatisfied and 3 being very satisfied. Complication scale (Table 3), where each patient was evaluated and given a score of 0 or 1 , with 1 indicating no complication and 0 indicating the occurrence of a complication. The Inter-rater reliability and correlation for photograph scale between the two surgeons was tested by Kappa and Pearson Correlation tests respectively (Tables 5,6,7).

The highest possible score is 38. A score of 3238 is considered excellent, 25-31 is considered very good, 18-24 was considered fair, and less than 18 is considered poor.

\section{RESULTS}

Eleven ears were affected; there were five females and five males, one of whom had bilateral affection. At the start of reconstruction, 9 patients were under the age of 10 (all with congenital defects) and 1 patient was between the ages of 20 and 30 .
The Mansoura ear reconstruction scale results were used to evaluate outcomes of reconstruction. We reported six patients who had excellent results, three patients who had very good results, and one patient who had a fair result. Table (4) shows the results.

Two patients had suffered from complications. First patient had a wound infection that was treated with antibiotics based on culture and sensitivity results. The cartilaginous framework was exposed as a result, and it was treated conservatively. $\mathrm{He}$ later developed a stitch sinus, which was removed with local anaesthesia. This patient went on to have a very good result. Second patient had an exposed tissue expander that was removed and then reinserted after 6 months of uneventful tissue expansion. He later developed an exposure of the cartilaginous framework, which necessitated the use of a local flap. This patient went on to have a fair result.

Table (1): Photograph scale.

\begin{tabular}{lcccc}
\hline & Poor & Fair & Very Good & Excellent \\
\hline Symmetry & 0 & 1 & 2 & 3 \\
Deformity & 0 & 1 & 2 & 3 \\
Elevation & 0 & 1 & 2 & 3 \\
Quality of details & 0 & 1 & 2 & 3 \\
Scars & 0 & 1 & 2 & 3 \\
\hline
\end{tabular}

Table (2): Patient satisfaction scale.

\begin{tabular}{lcccc}
\hline & Poor & Fair & Very Good & Excellent \\
\hline Symmetry & 0 & 1 & 2 & 3 \\
Deformity & 0 & 1 & 2 & 3 \\
Elevation & 0 & 1 & 2 & 3 \\
Quality of details & 0 & 1 & 2 & 3 \\
Scars & 0 & 1 & 2 & 3 \\
Repeated operations & 0 & 1 & 2 & 3 \\
\hline
\end{tabular}

Table (3): Complication scale.

\begin{tabular}{lcc}
\hline & Present & Absent \\
\hline Exposed cartilage & 0 & 1 \\
Skin disruption & 0 & 1 \\
Exposed expander/lost flap & 0 & 1 \\
Wound infection & 0 & 1 \\
Stitch Sinus & 0 & 1 \\
\hline
\end{tabular}


Table (4): Patient's data.

\begin{tabular}{|c|c|c|c|c|c|c|c|c|c|c|}
\hline Cases & 1 & 2 & 3 & 4 & 5 & 6 & 7 & 8 & 9 & 10 \\
\hline Age & 8 & 6 & 7 & 8 & 6 & 5 & 6 & 7 & 7 & 22 \\
\hline $\operatorname{Sex}$ & $\mathrm{F}$ & $\mathrm{M}$ & M & $\mathrm{F}$ & M & $\mathrm{F}$ & $\mathrm{F}$ & $\mathrm{F}$ & M & M \\
\hline Side affected & Rt & Rt & $\mathrm{Lt}$ & Rt & $\mathrm{Lt}$ & $\mathrm{Lt}$ & Rt & $\mathrm{Lt}$ & Rt & Bilateral \\
\hline Photograph scale score & 13.5 & 11 & 13.5 & 14 & 8 & 12.5 & 13.5 & 14 & 10.5 & 14 \\
\hline Patient Satisfaction score & 16 & 13 & 16 & 18 & 8 & 14 & 15 & 15 & 12 & 17 \\
\hline Complication score & 5 & 2 & 5 & 5 & 2 & 5 & 5 & 5 & 5 & 5 \\
\hline Total score & 34.5 & 26 & 34.5 & 37 & 18 & 31.5 & 33.5 & 34 & 27.5 & 36 \\
\hline Result & Excellent & $\begin{array}{l}\text { Very } \\
\text { Good }\end{array}$ & Excellent & Excellent & Fair & $\begin{array}{l}\text { Very } \\
\text { Good }\end{array}$ & Excellent & Excellent & $\begin{array}{l}\text { Very } \\
\text { Good }\end{array}$ & Excellent \\
\hline
\end{tabular}

F: Female. M: Male. Rt: Right. Lt: Left.

Table (5): Inter-rater reliability for photograph scale between the two assessing surgeons.

\begin{tabular}{llcl}
\hline & Value of Kappa & Standard error & $p^{*}$ \\
\hline Symmetry & 0.565 & 0.279 & 0.021 \\
Deformity & 0.655 & 0.196 & 0.01 \\
Elevation & 0.8 & 0.186 & 0.01 \\
Quality of details & 0.167 & 0.226 & 0.477 \\
Scars & 1 & 0 & 0.002 \\
\hline
\end{tabular}

$* p$-value significant $<0.05$.

*Correlation is significant at the 0.01 level (2-tailed).

Table (6): Correlation between total photograph score by the two assessing surgeons.

\begin{tabular}{ll}
\hline Pearson Correlation* & 0.792 \\
$p^{* *}$ & 0.006 \\
\hline
\end{tabular}

$* * p$-value significant $<0.05$.

Table (7): Difference between total photograph score by the two assessing surgeons

Wilcoxon Signed Rank Test $\quad p^{*}=0.885$

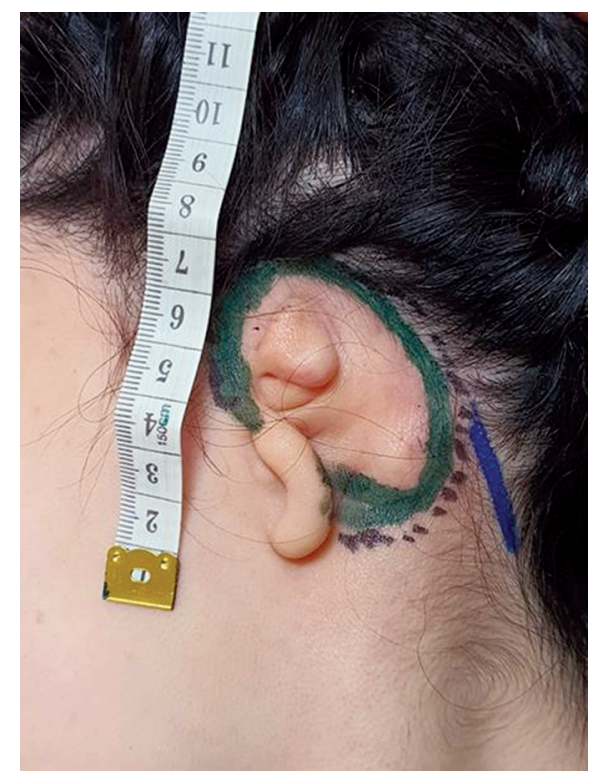

Fig. (1): Pre-operative marking, Blue line is planned skin incision, Black dotted line marks the hairline, Green line denotes planned skin pocket.

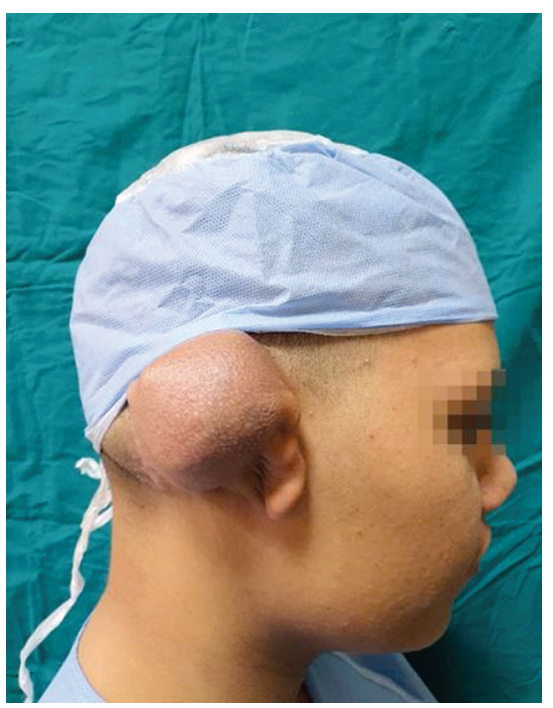

(A)

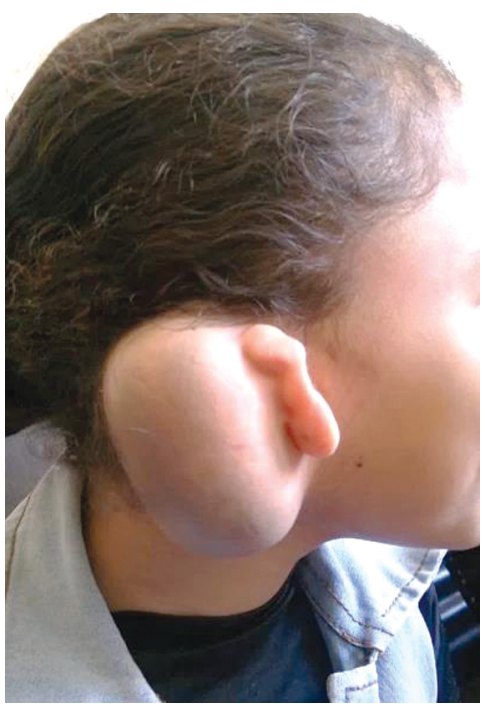

(B)

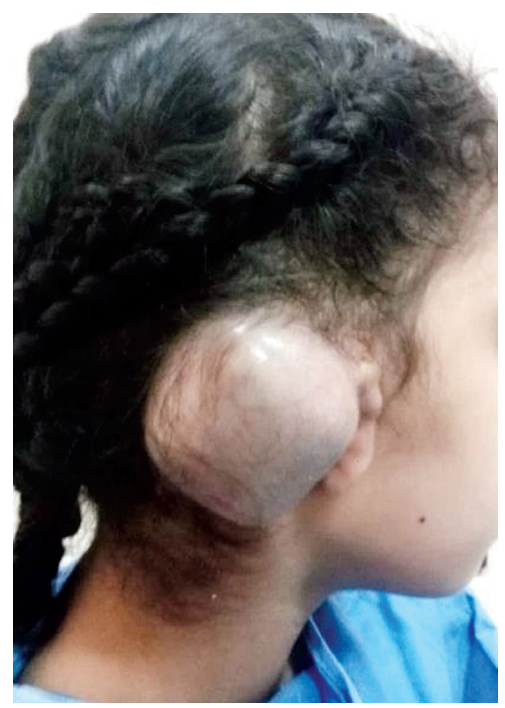

(C)

Fig. (2): (A,B,C) Showing tissue expander after inflation. 


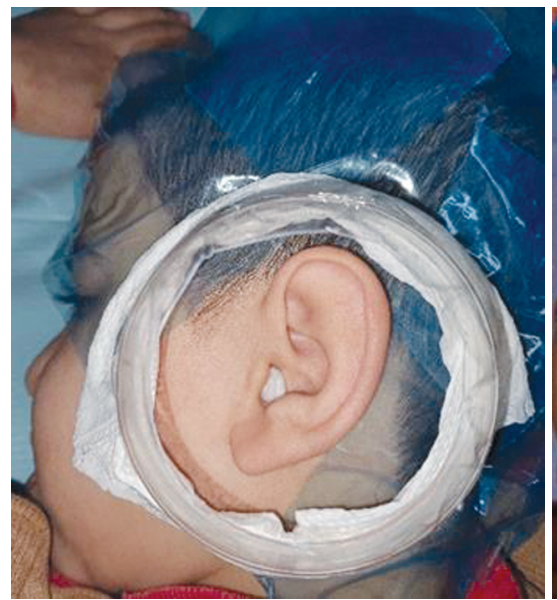

(A)

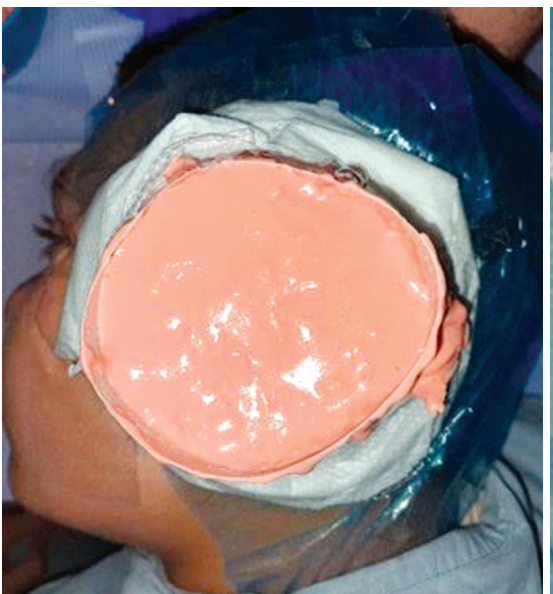

(B)

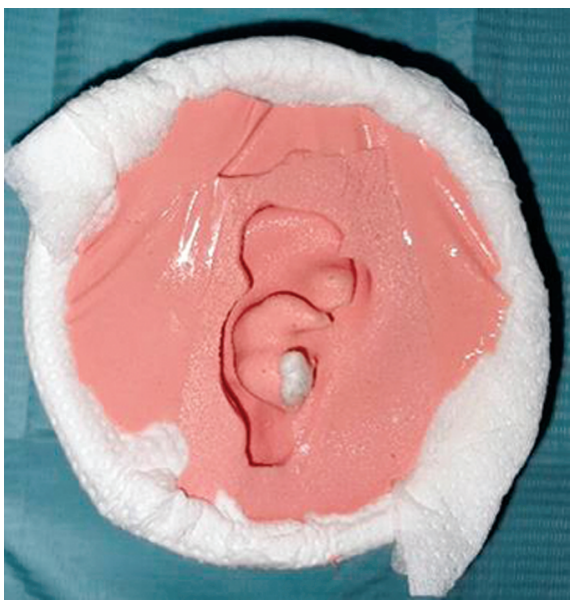

(C)

Fig. (3): (A) Plastic frame fixed to the normal auricle, (B) Alginate poured into frame, (C) Negative alginate imprint of normal auricle.

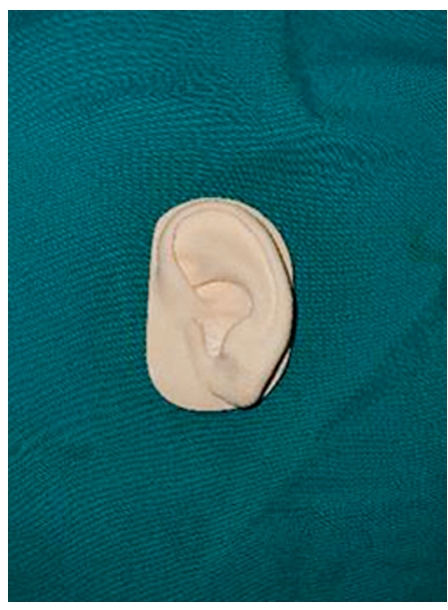

(A)

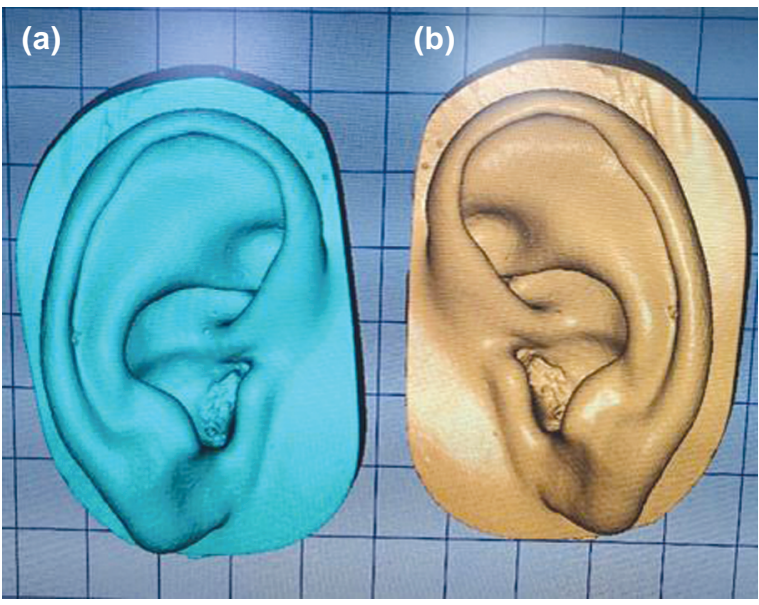

(B)

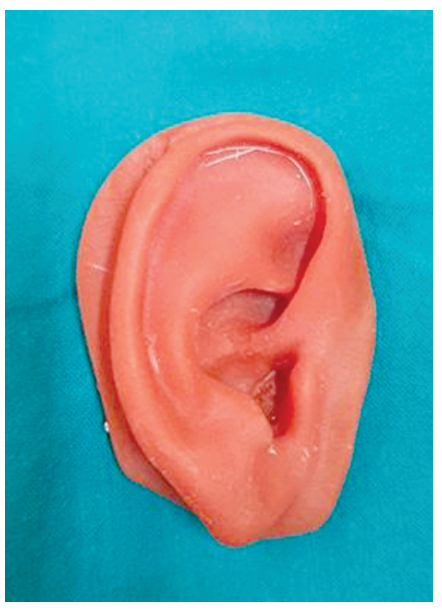

(C)

Fig. (4): (A) Gypsum model of normal auricle. (B) a) Showing render of auricle to be reconstructed after being flipped in CAD software, b) Showing scanned render of normal auricle. (C) 3D printed auricle.

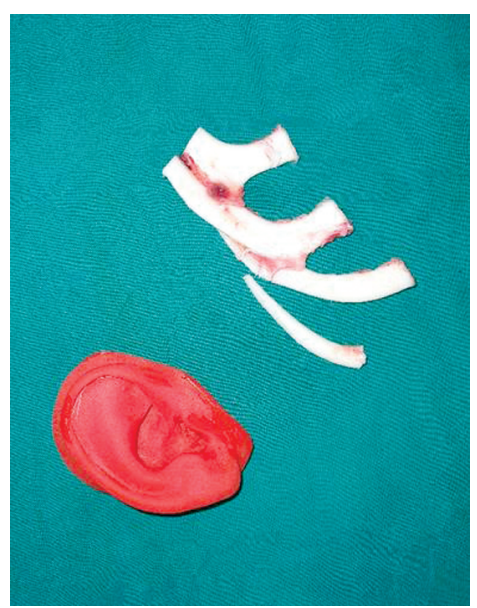

(A)

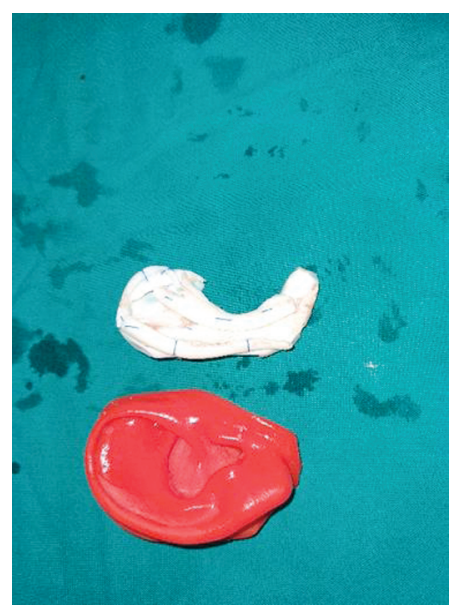

(B)

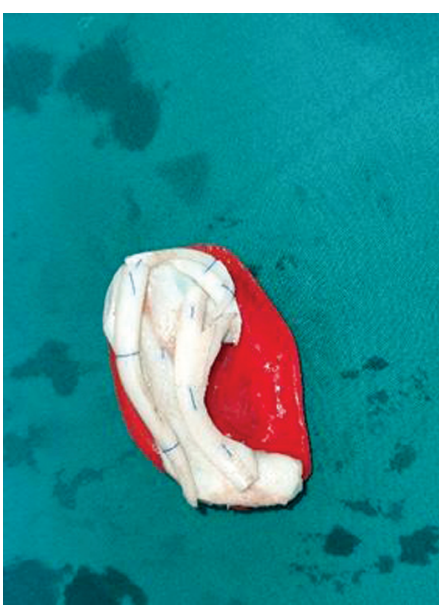

(C)

Fig. (5): (A) Harvested $6^{\text {th }}, 7^{\text {th }}, 8^{\text {th }}$, and $9^{\text {th }}$ costal cartilages with preservation of the synchondrosis, 3D template alongside harvested costal cartilage, $(\mathrm{B}, \mathrm{C}) 3 \mathrm{D}$ template alongside framework. 
Fig. (6): The reconstructed auricle after insertion of suction catheters and skin closure.
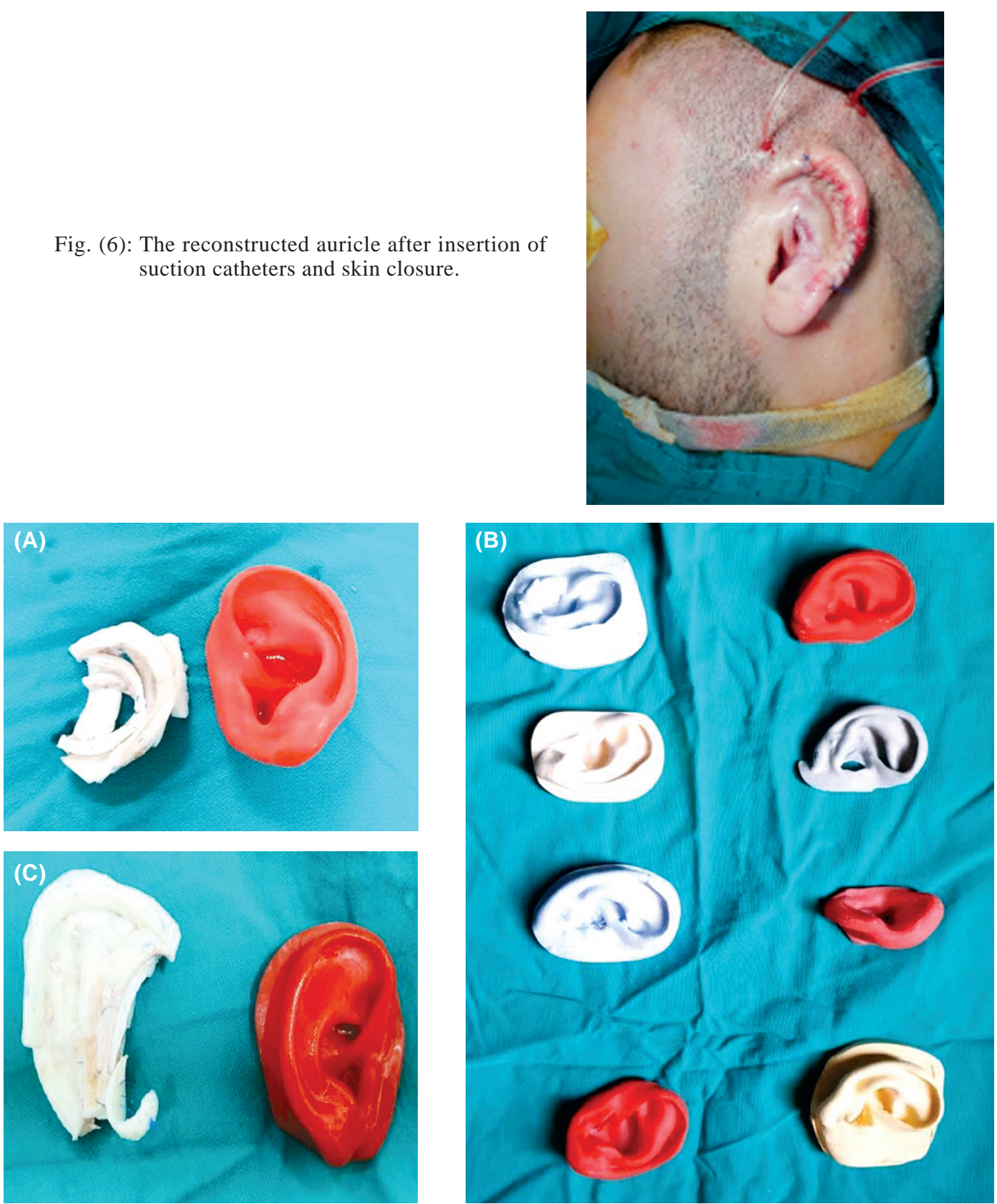

Fig. (7): Different shapes of 3D templates and printing and different shapes of cartilage framework.
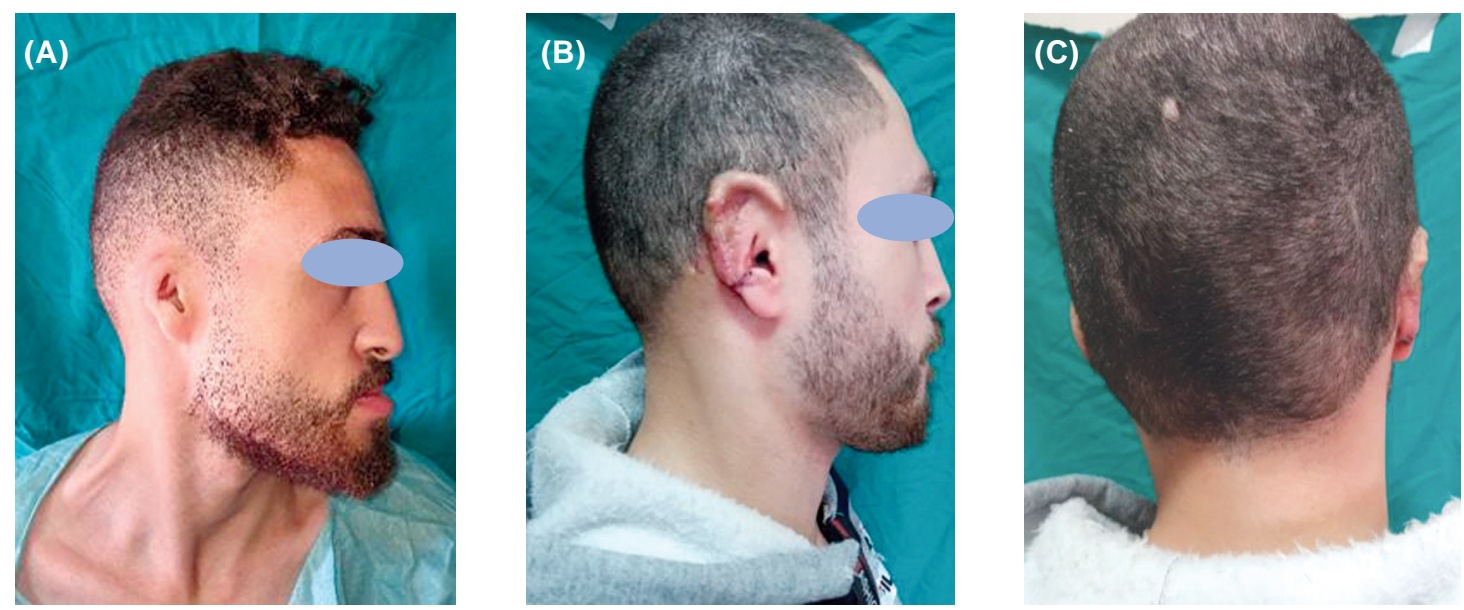

Fig. (8): Twenty two years old male with concha type microtia (A) Pre-operative left side. (B,C) Post-operative. 


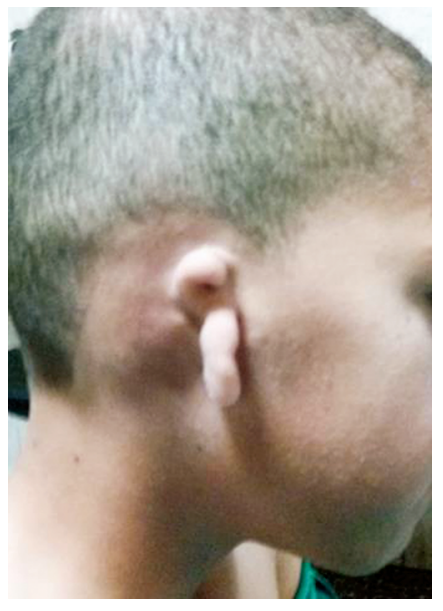

(A)

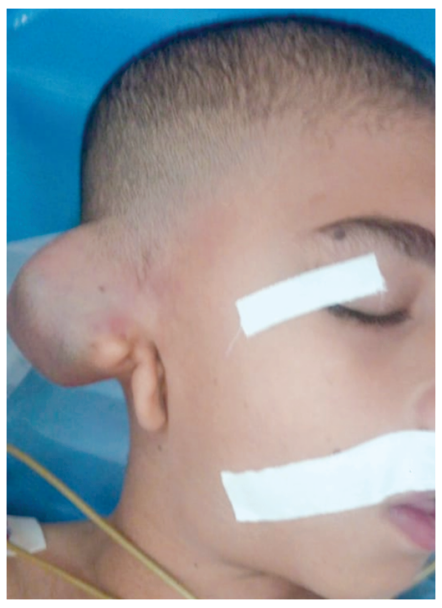

(B)

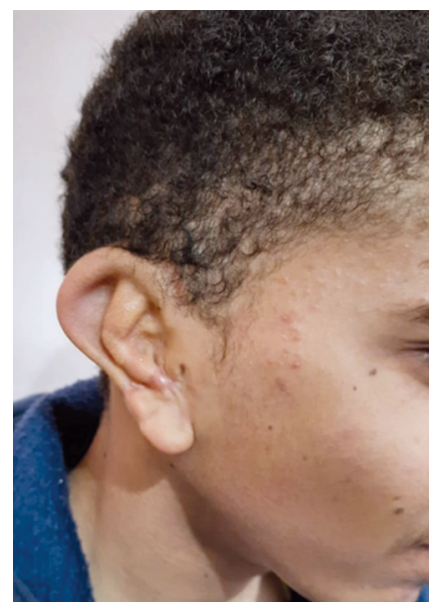

(C)

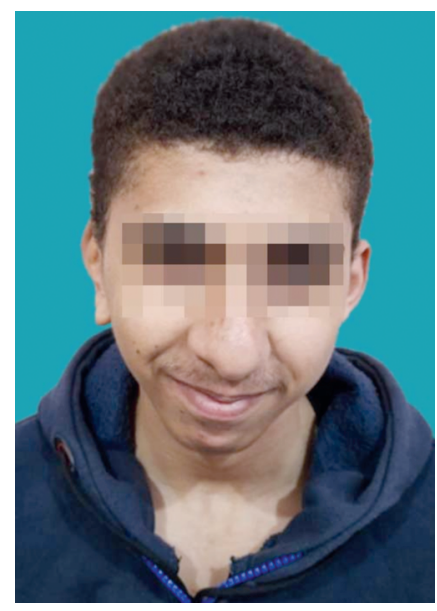

(D)

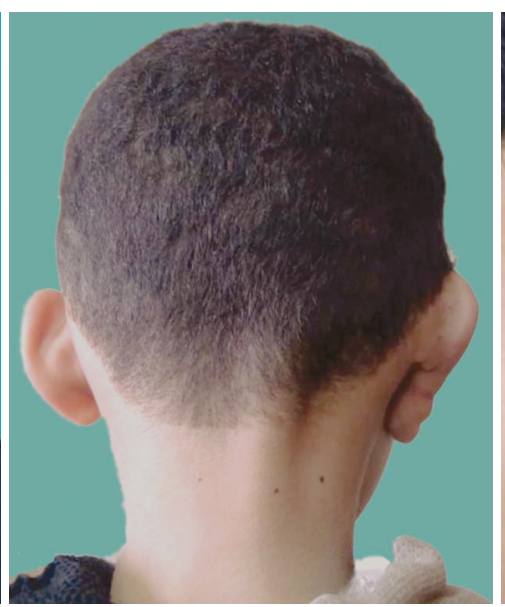

(E)

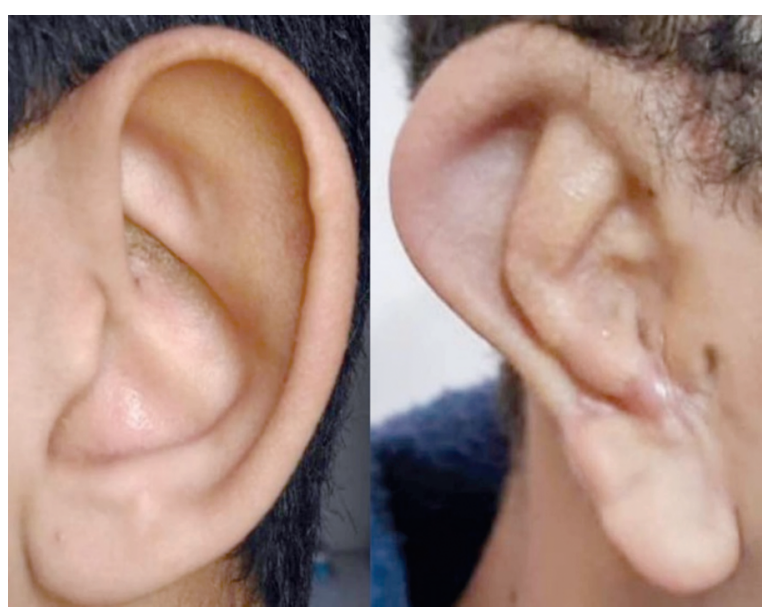

(F)

Fig. (9): Nine years old male with right sided microtia. (A) Pre-operative (B) Tissue expansion. (C,D,E,F) One-year postoperative.

\section{DISCUSSION}

The human ear with its position on the lateral aspect of the head and with its unique structure and complexities poses an intriguing challenge in its reconstruction for the plastic surgeon [1]. The normal auricle plays a unique aesthetic role, where even the smallest irregularity can stand out and cause a significant psychological burden for the patient [13].

Before the use of 3D auricular templates by Kelly and Kaneko in 1993, auricular templates were done by drawing of the outline of the contralateral ear on a plain X-ray film, or as done by Stotland and Chang, where they used a waterproof mirror image photograph of the contralateral ear $[14,15]$.

Brent used a tracing of the contralateral ear on a film, then flipping it and making smaller several millimeters in all directions to accommodate the extra skin thickness. The film was then taped on the side to be reconstructed and adjusted to achieve symmetry with the normal ear, and thus achieving the desired position [16].

Kaneko in 1993 first used a 3D measurement utilizing a laser slit lamp scanning system. The scan took 10 seconds, and it was reported that there was difficulty in stabilizing the patient's head. They also reported difficulties in scanning the posterior surface of the ear due to the narrow auriculocepahlic angle [14].

Kelley et al., in 1998 described using synthetic clay carved in the shape of a normal auricle, which was then placed in an oven to harden to obtain a $3 \mathrm{D}$ auricular model. The model was based on images and photographs of cartilage frameworks, which while giving good details, is not specific to the patient and may not provide a good match to the contralateral normal auricle [17].

Chen et al., in 2004 used a urethane resin poured into a negative silicon cast to obtain a 3D auricular 
model which was used as an intraoperative guide. While it gave excellent details and was fabricated in sizes ranging from $52 \mathrm{~mm}$ to $70 \mathrm{~mm}$, it was not individualized to each patient, and was thus just a generic model of an auricle with different sizes [18].

Bos et al., in 2015 described a parametric system to create patient specific ear models. Using a 3D soft tissue CT scan of 4 cadaveric ears, they obtained a set of data used to create a base model of an auricle. Using 3D modeling software, they managed to create a parametrically adjustable model. This was then adjusted for each desired auricular template. This was an overly complicated method and did not guarantee that the template would be identical to the contralateral auricle [19].

Fan et al., in 2019 used a soft tissue CT scan to digitally transpose the mirror imaged normal ear to reconstruct the affected ear, and then defined a mask along with the digitally transposed ear to identify the correct position of the reconstructed ear. This 3D template was then printed and used as an intraoperative guide. This was an overly complex method and required a high-resolution soft tissue CT scan with $3 \mathrm{D}$ reconstruction of the auricle [12].

In this current technique, the drawbacks in the previous methods could be overcome. The use of alginate to create a negative imprint gave good details of all structures of the ear including the posterior surface and the concha, and when the imprint was taken intraoperative there was no difficulty in keeping the patients head from moving. The subsequent use of gypsum and mirror imaging then printing the final imprint was less complex than Fan's method while giving the same desired result. The precise positioning of the ear was easy to determine using negative suction prior to inserting the final fabricated framework.

In current research, authors used a patient's specific 3D model as an accurate guide for creation of cartilaginous framework from the harvested costal cartilages (Fig. 7). This allowed precise measuring of the costal cartilages to be harvested and allowed us to perform precise intraoperative carving of the framework, giving a well-defined detailed reconstruction, avoiding any notches, and achieving good coaptation while also decreasing our operative time.

Zhang et al., operated on 350 microtic ears and reported skin flap necrosis in 13 patients and hypertrophic scars in 21 patients [20]. Sabbagh operated on 100 cases with microtia and reported 7 cases of skin necrosis and 7 cases of wire extrusion [21]. Jiang et al., operated on 3332 patients with microtia over a 10 -year period using tissue expanders. Their reported complications were 116 patients with hematoma (3.5\%), 111 with exposure of tissue expander (3.3\%), 20 with wound dehiscence $(0.6 \%), 28$ patients with skin necrosis $(0.8 \%)$, 10 patients with extrusion of the wire $(0.3 \%)$ and 210 patients with hypertrophic scar (6.3\%) [22].

We reported an acceptable overall complication rate with accepted and satisfactory final outcomes (Figs. 8,9), and our incidence rate was similar to other authors.

The lack of a standardized method for postoperative aesthetic evaluation of the results after auricular reconstruction is also a point which is worth studying and evaluating.

The small sample size and short period of follow-up are the study's limitations. One of the main strengths of this study is the Mansoura ear reconstruction scale, which gives better evaluation of outcomes. To our knowledge, there is no standardized method for evaluation of the aesthetic outcome of ear reconstruction. The authors established a score scale that is not dependent on surgeon's opinion of his own results, but it is dependent on two independent surgeons, and patient's own evaluation of his reconstructed ear and complications score system. This score scale could be applied to any ear reconstruction operations not only microtia reconstruction. The authors recommend this scoring system to be used by multiple surgeons and centers over a longer period to allow for precise judging of the score and finding any drawbacks for any future modifications of our score scale.

Conclusion: To conclude, the use of a 3D printed auricular model specific to each patient for microtia reconstruction led to excellent results and patient satisfaction after reconstruction.

\section{Funding: None.}

Conflict of interest: The authors declare that they have no conflict of interest to disclose.

Informed consent: Informed consent was obtained from the patient or their legal guardian. The study was approved by ethical committee of Institutional Review Board (IRB), Mansoura Faculty of Medicine (MS.20.03.1062). 


\section{REFERENCES}

1- Pham T.V., S.V. Early and S.S. Park: Surgery of the auricle. Facial Plastic Surgery, 19 (01): 053-074, 2003.

2- Bauer B.S.: Reconstruction of microtia. Plastic and Reconstructive Surgery, 124 (1): 14e-26e, 2009.

3- Paput L., F. Bánhidy and A.E. Czeizel: Distribution of associated component abnormalities in cases with unclassified multiple ("syndromic") anota/microtia. International journal of pediatric otorhinolaryngology, 75 (5): 639-647, 2011.

4- Cugno S. and N.W. Bulstrode: Bilateral autologous microtia reconstruction: A simultaneous two-stage approach. European Journal of Plastic Surgery, 39 (4): 257-264, 2016.

5- Lee K.T., et al.: Association of congenital microtia with environmental risk factors in South Korea. International journal of pediatric otorhinolaryngology, 76 (3): 357-361, 2012.

6- Wu J., et al.: Epidemiological analysis of microtia: a retrospective study in 345 patients in China. International journal of pediatric otorhinolaryngology, 74 (3): 275$278,2010$.

7- Griffin M., et al.: Comparison of the compressive mechanical properties of auricular and costal cartilage from patients with microtia. Journal of Biomechanics, 109688, 2020.

8- Cuccolo N.G., et al.: Reconstruction of Congenital Microtia and Anotia: Analysis of Practitioner Epidemiology and Postoperative Outcomes. Plastic and Reconstructive Surgery Global Open, 7 (6), 2019.

9- Zhang M., et al.: Investigation of Microsurgical Technique Combined with Skin Flap Expansion for Ear Reconstruction in Treating Hunter Type III Congenital Microtia. Annals of Plastic Surgery, 78 (6): 680-683, 2017.

10- Choi,J.W. and N. Kim: Clinical application of threedimensional printing technology in craniofacial plastic surgery. Archives of Plastic Surgery, 42 (3): 267, 2015.
11- Javaid M., et al.: Product design and development using polyjet rapid prototyping technology. Control Theory Inf, 5 (3): 12-20, 2015.

12- Fan X., et al.: Comparison of three-dimensional and twodimensional templates on auricle reconstruction in patients with unilateral microtia. American journal of translational research, 11 (6): 3771-3778, 2019.

13- Ghassemi A., et al.: Surgical management of auricular defect depending on the size, location, and tissue involved. Journal of Oral and Maxillofacial Surgery, 71 (8): e232e242, 2013.

14- Kaneko T.: A system for three-dimensional shape measurement and its application in microtia ear reconstruction. The Keio Journal of Medicine, 42 (1): 22-40, 1993.

15- Stotland M.A., W.T.J.P. Chang and R. Surgery: A better template for microtia reconstruction: the waterproof, mirror-image digital photograph of the contralateral ear, 119 (7): 2088-2091, 2007

16- Brent B.: Microtia repair with rib cartilage grafts: A review of personal experience with 1000 cases. Clin. Plast. Surg., 29 (2): 257-71, 2002.

17- Kelley T.F., et al.: The use of 3-dimensional models in auricular reconstruction, 124 (3): 335-338, 1998.

18- Chen Z.-C., et al.: Microtia reconstruction with adjuvant 3-dimensional template model, 53 (3): 282-287, 2004.

19- Bos E., et al.: Developing a parametric ear model for auricular reconstruction: A new step towards patientspecific implants, 43 (3): 390-395, 2015.

20- Zhang Q., et al.: Auricular reconstruction for microtia: Personal 6-year experience based on 350 microtia ear reconstructions in China, 123 (3): 849-858, 2009.

21- Sabbagh W.: Early experience in microtia reconstruction: The first 100 cases. Journal of Plastic, Reconstructive \& Aesthetic Surgery, 64 (4): 452-458, 2011.

22- Jiang H., et al.: Ten-year experience in microtia reconstruction using tissue expander and autogenous cartilage, 72 (8): 1251-1259, 2008. 